\title{
Modal Propellant Gauging in Microgravity
}

\author{
Taylor Peterson ${ }^{1}$, Cassandra Bossong ${ }^{1}$, and Bennett Bartel ${ }^{1}$ \\ ${ }^{1}$ Student (Physics, Carthage College) \\ Kenosha, WI, United States
}

\begin{abstract}
The Modal Propellant Gauging (MPG) team consists of multidisciplinary undergraduate researchers from Carthage College. This continuously changing team has been developing and testing a fuel gauging system for use in microgravity environments since 2008. Using Experimental Modal Analysis (EMA) techniques, the goal of the project is to develop a flight-ready fuel gauging technology by correlating the modal response of a 1-g surface to the microgravity surface response at the same fluid fill fraction. The technology has been integrated into a manned parabolic flight payload and a Blue Origin New Shepard sub-orbital flight payload. The parabolic flight payload contains two propellant tanks and is designed to measure the modal response of each propellant tank to an injected white noise signal via piezoelectric sensors. Flight data shows that the MPG method can measure fuel with greater than or equal to $1 \%$ resolution at and below 50\% fill levels. Similarly, the Blue Origin payload also measures the modal responses of the propellant tanks, however it has an extended period of time in microgravity. Under funding from the Wisconsin Space Grant Consortium during the summer of 2019, the MPG team made improvements to the Blue Origin New Shepard research flight payload which has seen flight on the New Shepard vehicle in January of 2019 and is manifested to fly again in an upcoming launch.
\end{abstract}

\section{Introduction}

The Modal Propellant Gauging (MPG) project aims to develop a higher resolution and light-weight fuel gauging method for microgravity environments compared to traditional methods. MPG is a student led project with the guidance and aid of Dr. Kevin Crosby. The MPG project has two payloads integrating this technology: one of which is for manned parabolic flights; the other is a

sub-orbital payload that has seen flight on Blue Origin's New Shepard Mission in January of 2019 and is manifested to fly once more on an upcoming launch. The purpose of the sub-orbital payload is to determine the settling time of the liquid in a longer zero gravity duration compared to the parabolic flight payload.

1.1 Microgravity Team Undergraduate students at Carthage College from an abundance of backgrounds have come together to develop the MPG technology since 2008. Every March and November the MPG students fly with their payload and advisor Dr. Kevin Crosby on parabolic flights through the Zero-G Corporation, previously with NASA until their program was discontinued. NASA has funded multiple iterations of the MPG project, all of which were advised by Dr. Crosby. These projects give students valuable hands on experience with space technology development through partnership with NASA and other academic researchers around the world. It is essentially graduate level research in an undergraduate setting.

1.2 Purpose For the past 50 years, inaccurate fuel gauging has been a large problem in the space industry, dating back to the Apollo 11 moon landing. As NASA aims to head back to the Moon and colonize its surface, this problem must be addressed. For human spaceflight, certification of spacecraft requires higher gauging accuracy than is currently possible. Propellant mass gauging is seen as a critical path technology for sustained human presence in space. The Modal Propellant 
Gauging technology is a candidate to amend this long-lived problem. MPG is a lightweight, noninvasive method of accurately measuring the amount of propellant in fuel tanks in microgravity environments. This technology has been developed to be used on the Orion Spacecraft, which will be the transportation between the Earth and the Moon in preparation of the lunar orbiting station, Gateway. Gateway will serve as a temporary home for astronauts, and will include living quarters, labs for research, docking parts for visiting spacecrafts, and more. Gateway will have decent and ascent modules to get to and from the surface of the Moon.

The lifespan of spacecraft depends highly on propellant. In the majority of spacecrafts, over $80 \%$ of the spacecrafts mass is propellant. It costs about $\$ 10,000$ to put a single pound of payload into Earth's orbit. Reducing launch mass is an essential design specification for low-gravity fuel gauging. This would extend mission lifetime and be in agreement with international requirements of end-of-life deployment for satellites heading to "graveyard orbits". Traditional fuel gauging methods, such as pressurization, are rendered useless in microgravity environments. Currently, as the fuel in propellant tanks deplete, the system becomes less and less accurate, when it matters the most. The MPG approach has a higher accuracy as the fluid in the tank gets lower.

MPG is a collaboration between Carthage Space Sciences, the advanced Exploration Systems Engineering Division at NASA Kennedy, and the Propulsion Center at NASA Johnson. It has been funded by the Wisconsin Space Grant Consortium.

1.3 Concept The Modal Propellant Gauging (MPG) technology analyzes resonance frequencies of fluid-filled propellant tanks. Liquid in microgravity environments does not settle than same as in a 1-g equilibrium state on Earth. In low gravity, propellant clings to the inner walls of the tanks due to high surface tension and capillary flow. This is called "mass-loading" and the MPG technology uses it to determine the fluid fill-fraction inside the propellant tanks.

MPG uses Experimental Modal Analysis (EMA), a widely used technique that characterises an objects structure in terms of its natural characteristics, including resonant frequencies. Every object has a set of natural frequencies and modes determines by material properties, geometries, and mass. In propellant tanks, as the fluid depletes, the responsive frequency increases. MPG focuses on responsive frequencies ranging from $500 \mathrm{~Hz}$ to $1500 \mathrm{~Hz}$. The analysis does not go any higher due to an increase in electrical noise. This technique works best at fluid levels under about $50 \%$ since anything above will coat the inner tank walls with fluid.

The EMA technique requires we apply a broadband of white noise frequencies from $100 \mathrm{~Hz}$ to $10 \mathrm{kHz}$ to stimulate the structural acoustic resonance in the model propellant tanks. The white noise is applied using piezoelectric (PZT) transducer patches and recorded using PZT sensors on discrete locations on the outer tank walls. Using Fast Fourier Transform (FFT), we compute a frequency response function (FRF) from the vibration responses at the different locations by taking the ratio of the input signal and the output response. The input sensor is directly next to the transducer patch as a reference signal, referred to as the "monitor". The output is placed away from the transducer and the monitor, recording the amplified resonant frequencies of the model tanks, referred to as the "sensor". The resulting FRF is a complex valued function, though the system we are modeling is 
lightly dampened, allowing for clear peaks that represent the response frequencies, as seen in Figure 1.

\section{Parabolic Flights}

To test the validity and accuracy of the technique in microgravity environments, the MPG payload has been manifested to fly in a modified Boeing-727, named G-FORCE ONE, with parabolic flight trajectories that simulate microgravity during the free-fall portion of the parabola. Each parabola allows data to be taken for nearly 20 seconds of zero gravity at a time. Following each portion of microgravity is a period of $2 g$ as the aircraft pulls up to climb the height it fell through. The current Carthage College MPG Team has been selected for funding to fly on multiple parabolic flight campaigns.

2.1 Experimental Flight Payload The experimental flight payload can be seen in Figure 2 . The mechanical frame of the payload was constructed using various different aluminum 80/20 tslots. Secondary containment is restrained by gaskets holding in clear polycarbonate panels on all sides to view inside of the payload. The top of the payload is connected via hinges, allowing access into the inside of the payload. Inside secondary containment, there are two acrylic tanks connected via fluid flow loops allowing for automatic liquid drain and fill. On each outer side of the tanks there is a liquid fluid-level gauge for visual confirmation of the fill fraction. There are four PZT sensors adhered to the outer walls of each tank; one transducer patch, two sensors, and one monitor.

Data acquisition, signal control, and flow loop control are all maintained by a National Instruments 9133 data acquisition (DAQ) system. The DAQ is mounted in the interior of the payload in between the two modeled propellant tanks. The DAQ and flow loops are controlled using an industrial grade monitor, mounted on top of the payload exterior. The monitor communicates to the DAQ through LabVIEW. The electronics for the entire payload are also located on the exterior top of the structure, containing all necessary components to power each system.

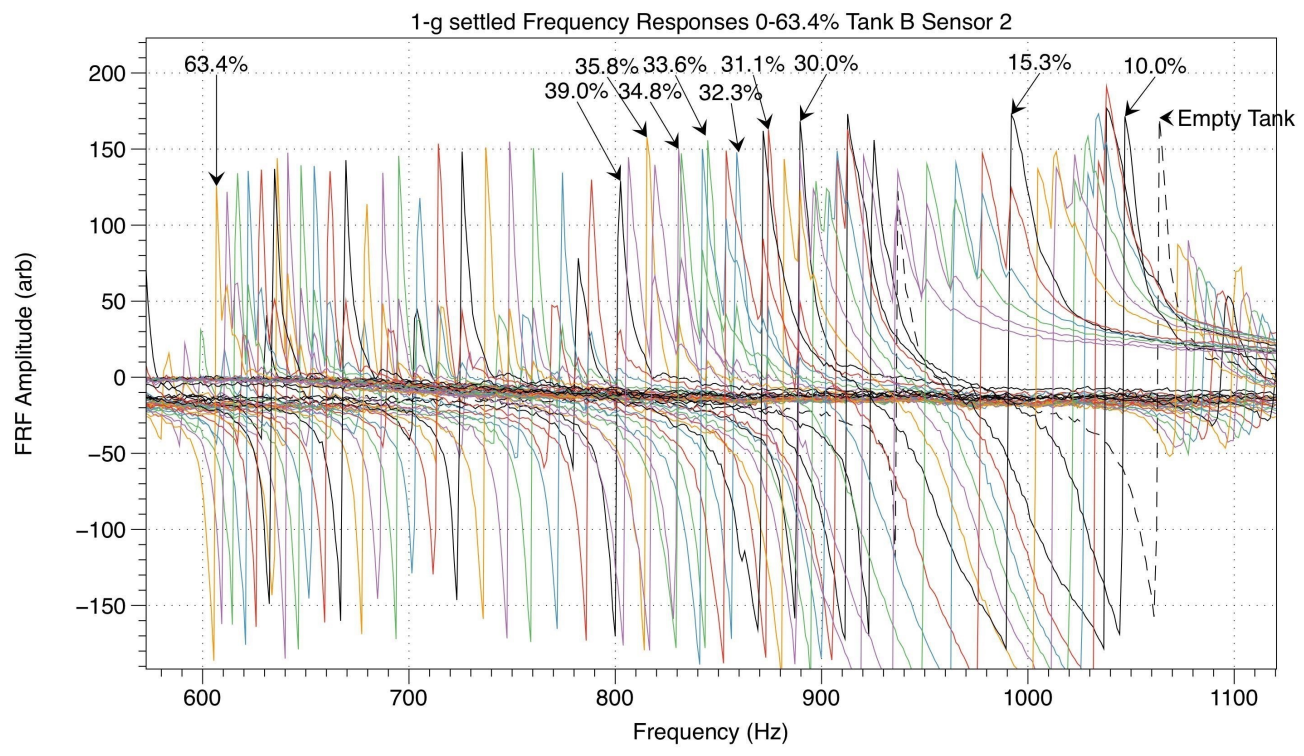

Figure 1: Frequency responses of a $1 \mathrm{~g}$ equilibrium surface 
2.2 Data collection and Analysis Due to the payload being manned, the data collection process during flight is simple. The flight crew informs the passengers when the aircraft is "pushing over," which signifies the start of the zero-g period. The crew then activates the software by pushing a button on the touchscreen monitor to record data for the brief period of zero gravity. However, the zero-g portions are not perfect due to the negative impulses that cause the liquid to slosh around in the tanks. Our solution to this issue is to average the data for the same fill levels. Every 5 parabolas the fill level is changed during a 1-g "straight and level" period, and then testing resumes for the next set of parabolas. This extra sloshing does not allow the liquid inside the tanks to fully settle as desired. Due to this we have implemented three concentric rings attached to the inner walls of the tanks in attempt to get more realistic results.

Figure 1 shows a sample of data collected from a single tank in 1-g over a range of an empty tank to fill level of roughly $50 \%$ of the tank's volume. The technique is able to recognize a one percent change of total volume based on the modal response of the tank. This data was taken in our lab; while aboard the aircraft there is substantial noise in the data due to fluid sloshing which decreases the resolution.

\section{New Shepard Payload}

The Modal Propellant Gauging technique has proven to work multiple times and cuts down on costs due to the lightweight nature of the sensors. NASA has shown great interest in implementing the MPG technology for future missions to Mars. The main concern NASA expressed is how long it takes the liquid to reach equilibrium and if it would happen in a different configuration each time. We have done a plethora of Computational Fluid Dynamics (CFD) simulations, which show that this will not occur. The liquid should coat the walls of the tanks evenly every time. The parabolic flights allow for about 22 seconds in microgravity, this is not a long enough time to watch the liquid settle and we occasionally experience negative gravity, causing the liquid to slosh rapidly. The CFD simulations were tested at different forces and velocities and show that the liquid should settle in about 90 seconds. In order to test MPG for a longer duration, a smaller version of the payload has been selected to take flight on Blue Origin's New Shepard Vehicle. This allows for 3 minutes in pure zero gravity, which should alleviate NASA's concerns with the technique by providing footage of how the liquid will settle in the tanks.

Blue Origin's New Shepard Vehicle is a re-usable, sub-orbital launch vehicle designed to transport a six-person crew to the region beyond Earth's lower atmosphere. The research flight our payload will fly aboard is an unmanned flight, and the crew capsule will contain stacks of payload lockers in place of seats to hold a handful of experiments on each flight. 


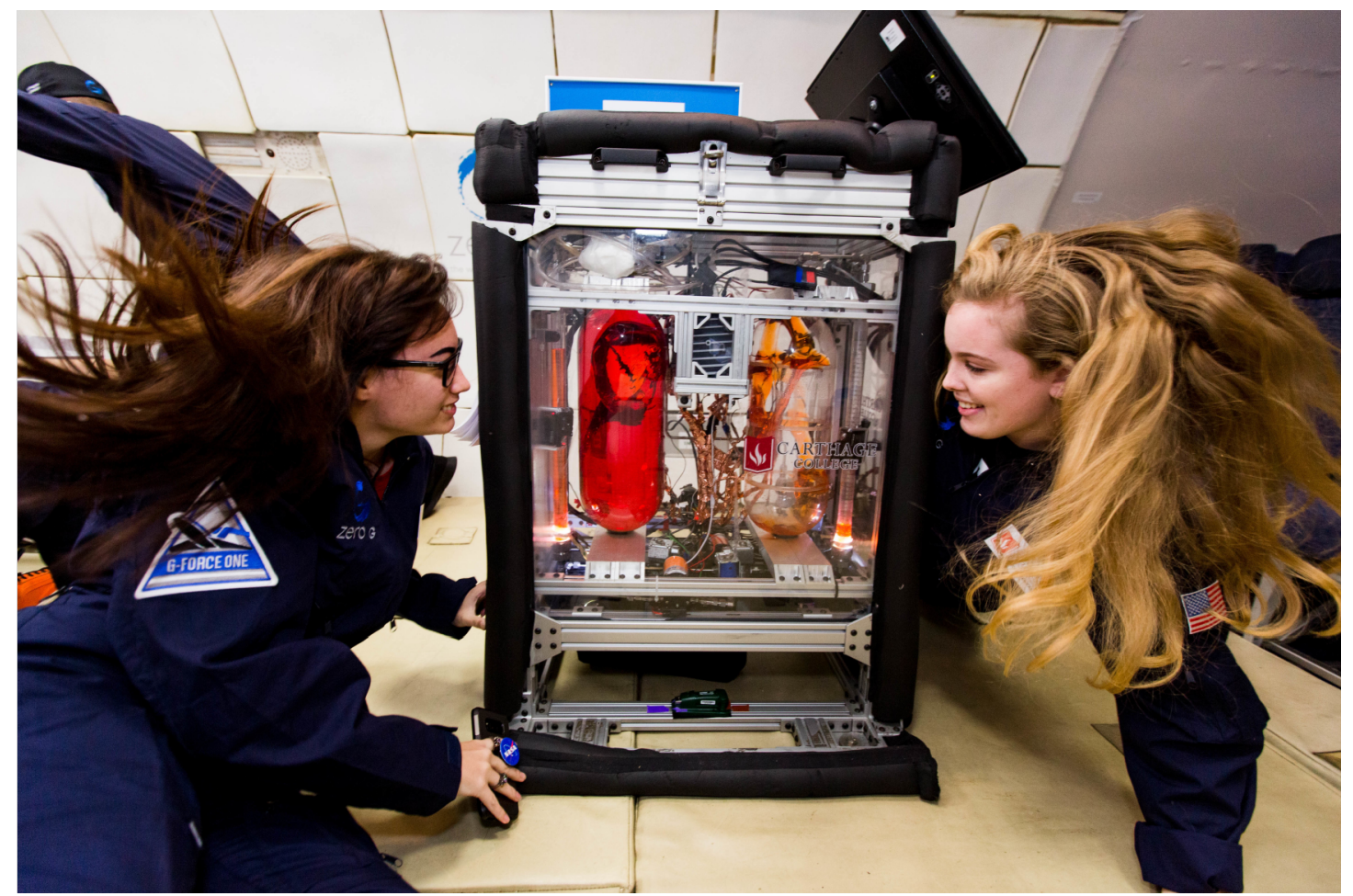

Figure 2: Taylor Peterson (left) and Celestine Ananda (right) with the Parabolic flight payload on-board GFORCE ONE

3.1 Payload Concept The MPG team designed this payload starting with the propellant tanks and moving outward, deciding on cylindrical tanks. Since fluid properties do not scale linearly, careful consideration was given when deciding the size of the tanks, keeping the dimensional restraints of the locker in mind. If the propellant tanks were too small, the properties of the fluid and the fluid dynamics, would decrease the chances of accurately recreating the flight conditions. In contrast, if the tanks were too large, the number of propellant tanks contained within the payload must decrease, which reduces the amount of data we would record, including the different volumes of fluid in each tank. Furthermore, it was important to consider not only the absolute dimensions of the tanks, but the ratio of the height of the tanks to their diameter. After running various Computational Fluid Dynamics (CFD) simulations, the dimensions of the tanks were chosen to be 10.8 " in height and 6" in diameter. Given the size of the propellant tanks, water will be used as the model propellant to preserve the scaling properties of the fluid dynamics. In microgravity environments, the liquid behavior of water and propellant are almost identical. The remaining elements of the payload were designed around the size and arrangement of the tanks, and the overall structure was designed to use all of the available interior volume provided by the payload locker.

Unlike the parabolic flight payload, the New Shepard payload will use only one sensor compared to the two sensors from the parabolic flight payload. In addition, there is no flow loop in the New Shepard payload, so each tank will contain various fixed fill levels. Lastly, the experiment will run autonomously, collecting data throughout the entire duration of the flight. Overall, the payload was designed to be as simple as possible in mechanical and electrical design, as well as the concept. 
The Modal Propellant Gauging team decided to build a simplistic design for the payload to increase the chances of a successful mission.

3.2 Payload Design and Construction The payload was made a rectangular cube of nominal dimensions $18 " \times 14 " \times 18$ ". The structural frame of the payload was constructed using 10 -series $80 / 20$ aluminum t-slots, and the t-slots are held together by $80 / 20$ aluminum joining plates that use bolts and t-nuts. A labeled diagram of the payload can be found in Figure 3.

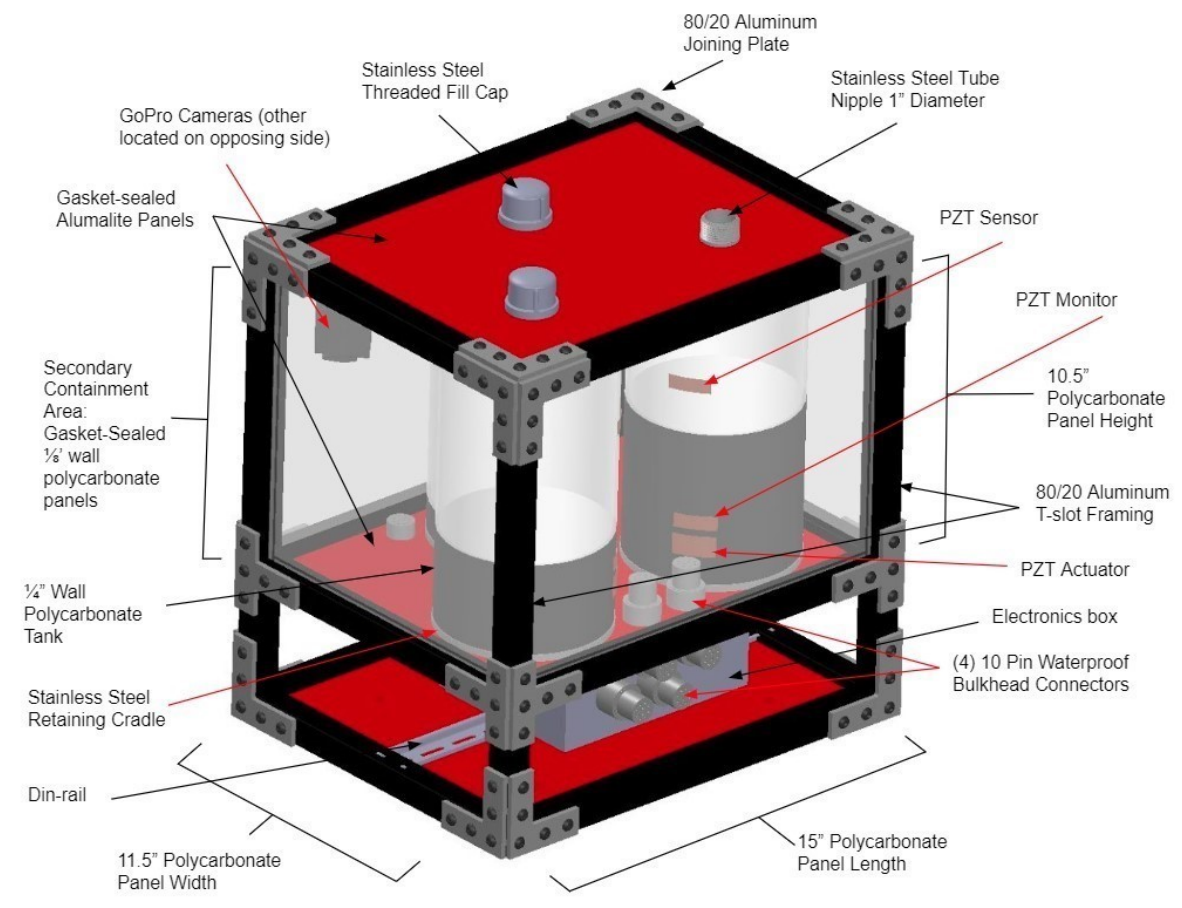

Figure 3: Labeled SOLIDWORKS model of New Shepard payload

3.3 Secondary Containment The Secondary containment is sealed via four gasketed polycarbonate panels on each side, as well as two gasketed Alumalite panels located at the top and bottom of the payload. At the top of the Alumalite panels, there are three threaded stainless-steel pipe nipples that are sealed. These allow external access to the inside of the tanks, however, there is not access to secondary containment from these points. Within this secondary containment, there are three cylindrical propellant tanks arranged in an isosceles triangular formation, which are sealed at the top by means of three mounting caps. In the top corners of secondary containment, there are two GoPro cameras angled downward at approximately 45-degrees in order to optimize the view of the three propellant tanks. This is essential for the mission because it provides in-flight footage showing not only the liquid behavior in microgravity, but also the settle time and the settle state of the fluids. In order to provide better lighting for the cameras, there are three LED lighting panels located on the outside of secondary containment that will illuminate the tanks during the flight with Blue Origin. 
3.4 Electronics and Mounting Bay The electronics box holds the data acquisition system, power regulators, white noise generators, and the signal amplifiers. This box is made out of an aluminium box, and it is mounted to the payload deck through a use of a din rail. All of the required electrical connections are wired by four air-tight 10-pin connectors, which are transferred from secondary containment to the electronics box. The electronics box will be mounted to the payload locker by eight bolts going directly through the electronics and mounting deck, corresponding with the threaded holes located on the payload locker's pattern.

3.5 Design and Construction The construction of this payload was originally put together using SOLIDWORKS in order to verify the dimensions and prove the general concept. Following the final design, the process of building the payload began. Right before the launch in early 2019, construction of the payload was completed. This includes a full systems integration, completed flight simulations, and the integration of the payload design to cooperate with the payload locker. Figure 4 displays a labeled CAD model of the payload.

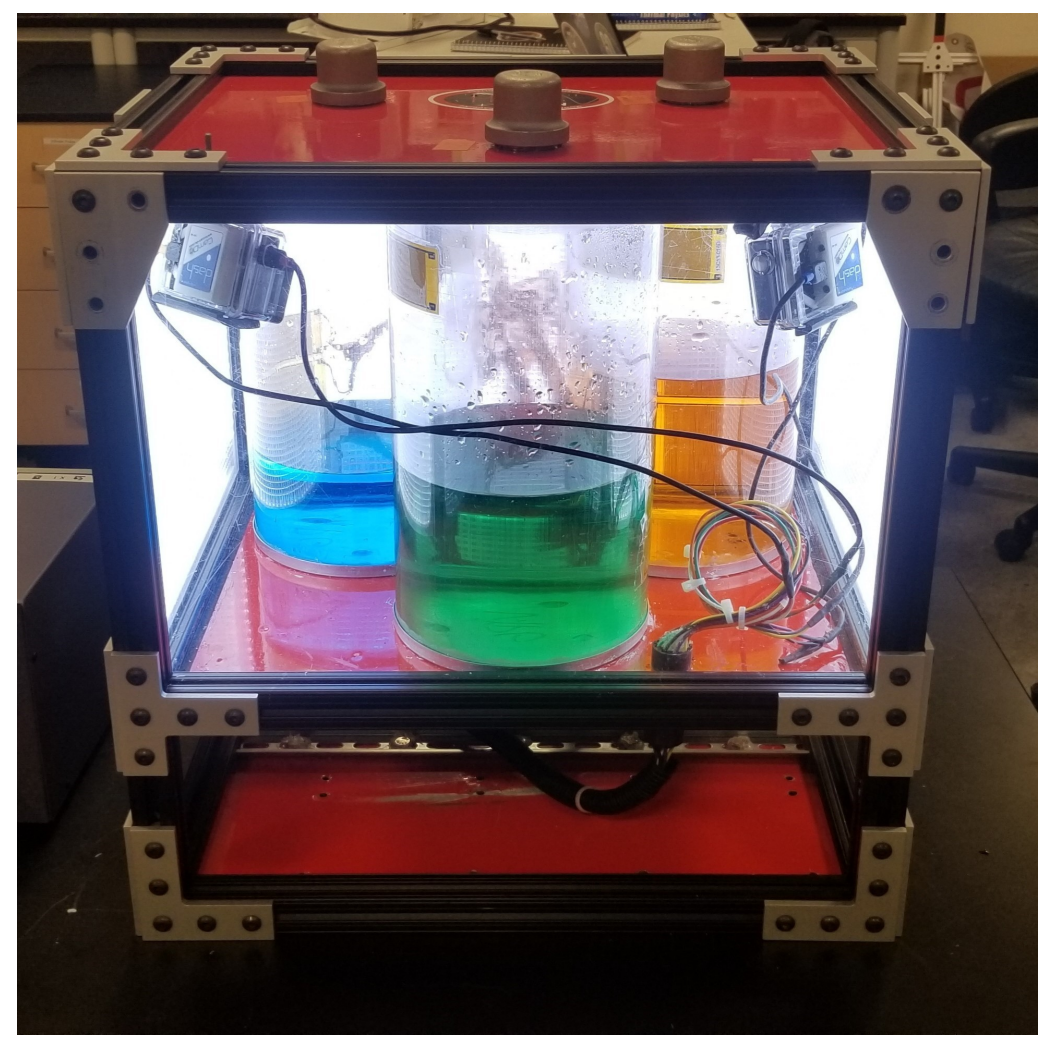

Figure 4: New Shepard payload

\section{Successful January Launch}

In January 2019, three research students on the Modal Propellant Gauging team - Taylor Peterson '21, Nicholas Bartel '20, and Celestine Ananda '20 - traveled down to Blue Origin's remote West Texas Launch Site to participate in a launch of the New Shepard Vehicle. The students had the opportunity to work with Blue Origin directly to prepare their payload to take flight on New Shepard. The payload successfully captured video of the propellant tanks throughout the duration 
of the mission. This footage shows that the time for the fluid to reach equilibrium is approximately 18 seconds, a much shorter time than estimated by our simulations. The team is now making improvements to their payload for a second upcoming launch.

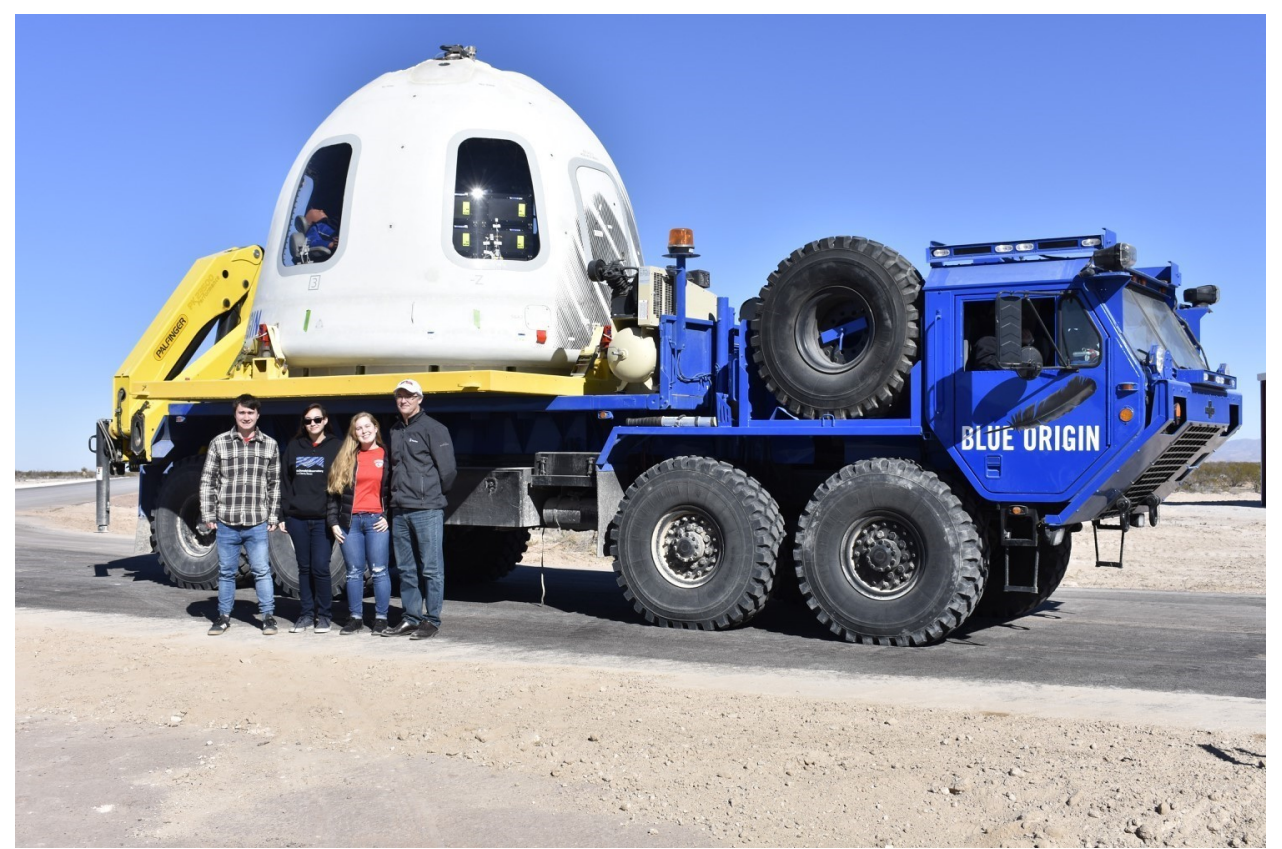

Figure 5: Research students and their mentor with the Crew Capsule after launch (from left to right - Nicholas Bartel, Taylor Peterson, Celestine Ananda, Dr. Kevin Crosby)

\section{Summary}

The Modal Propellant Gauging project is a collaboration of the Carthage College Space Sciences program and the Advanced Exploration Systems Engineering Division at Kennedy Space Center. The goal of this project is to develop a high-resolution, lightweight fuel gauging technology that determines the liquid behavior and fluid levels of propellant tanks. This technology will be implemented on the Orion/SLS Exploration Mission architecture in the Orion Service Module on the two propellant tanks. One concern with this technique is the off chance that the fluid may not reach the same equilibrium configuration every time. This would depreciate the accuracy of the system; however, our simulations show that this will not occur. In order to alleviate this concern, a smaller version of Modal Propellant Gauging was built to be tested on Blue Origin's New Shepard vehicle in January of 2019 and soon December of 2019. This payload allows an extended amount of time in microgravity, giving the liquid more than enough time to reach equilibrium. The smaller payload is helping in the act of finalizing the implementation of the MPG technique on the Orion/SLS Exploration Mission.

\section{Acknowledgments}

We would like to thank our advisor and the director of the Wisconsin Space Grant Consortium, Dr. Kevin Crosby, for giving us the amazing opportunity to work on this project. We would additionally like to thank the Wisconsin Space Grant Consortium for funding this project. Finally, 
we would like to thank the Zero-G corporation and Blue Origin for allowing us the opportunity to test our project in microgravity environments.

\section{References}

Avitabile, Peter. "Experimental Modal Analysis." Modal Analysis and Controls Laboratory, University of Massachusetts Lowell. 2001, http://innomet.ttu.ee/martin/MER0070/WB/WS9/WS9 Bell/Modal Analysis.pdf.

Crosby, K., Rundle, T., LeCaptain, K., Werlink, R. "Modal Propellant Gauging in Low Gravity.” National Center for Biotechnology Information. September 13, 2016, https://www.ncbi.nlm.nih.gov/pmc/articles/PMC6427914/.

Dunbar, Brian. "Moon to Mars Overview." National Aeronautics and Space Administration. April 5, 2019, https://www. nasa.gov/topics/moon-to-mars/overview.

Dunbar, Brian. "NASA's Lunar Outpost will Extend Human Presence in Deep Space." National Aeronautics and Space Administration. October 22, 2018, https://www.nasa.gov/feature/nasa-s-lunar-outpostwillextendhumanpresenceindeep-space.

Kauderer, Amiko. "The Tyranny of the Rocket Equation." National Aeronautics and Space Administration. May 1, 2012, https://www.nasa.gov/mission pages/station/expeditions/expedition30/tryanny.html.

National Aeronautics and Space Administration, Johnson Space Center. "Apollo 11 Mission Report November 1969." NASA. November 1969, www.nasa.gov/specials/apollo50th/pdf/A11 MissionReport.pdf. 